\title{
TRICHOPHYTON KURYANGEI n. sp. NOUVEAU DERMATOPHYTE AFRICAIN
}

\section{Par R. VANBReuseghem $\left({ }^{*}\right)$ et S. A. ROSEnTHAL $\left({ }^{* *}\right)$}

Au cours d'un examen qu'un d'entre nous (Vanbreuseghem 1957) fit en 1956 au Congo Belge et au Ruanda Urundi de 15.000 indigènes - adultes et enfants -, il isola, de quelque 500 prélèvements effectués sur le cuir chevelu des teigneux et qui donnèrent lieu, pratiquement tous, à des cultures de dermatophytes, huit souches qui ne répondaient pas aux descriptions classiques. Ces huit souches furent toutes isolées du Ruanda Urundi. L'enquête portant sur cette région a fait l'objet d'une publication (Vanbreuseghem 1961). Elle indique que les espèces de dermatophytes isolées furent: Trichophyton violaceum (68), Trichophyton ferrugineum (8), Trichophyton (langeronia) soudanense (1), Microsporum (Sabouraudites) langeroni (15) et un autre dermatophyte nommé provisoirement : Trichophyton kuryangei (8). C'est de ce champignon que traite le présent travail.

Des huit souches isolées, cinq provenaient du village de Kuryange situé à une altitude d'environ $1.500 \mathrm{~m}$., à $18 \mathrm{~km}$. au Nord d'Usumbura. Deux autres venaient du village de Sororezo (à 1.100 ou 1.500 mètres d'altitude, à quelques kilomètres au Nord d'Usumbura) et la dernière d'Usumbura même, au bord du Lac Tanganyka, soit à une altitude de $772 \mathrm{~m}$. Dans trois cas, $T$. violaceum, très répandu au Ruanda Urundi puisqu'il représente $75 \%$ des souches isolées par Vanbreuseghem, fut isolé en même temps que $T$. kuryangei.

Voici quelques renseignements cliniques sur les malades dont furent isolées les souches. Il s'agit toujours d'indigènes de race noire, atteints de teigne du cuir chevelu, ce qui n'exclut pas la possibilité de lésions sur le reste du corps que nous n'avons pas examiné. Les cheveux cassés, mesurés dans les prélèvements, n'attei-

(*) Département de Mycologie de l'Institut de Médecine Tropicale, Anvers (Belgique).

(**) Skin and Cancer Unit, New-York University, Bellevue Medical Center of New-York University, University Hospital, 330, Second Avenue, New-York, 3 (Etats-Unis).

Ann. de Parasitologie, T. XXXVI, N ${ }^{\circ} 5-6 .-1961$. 
gnaient pas plus de 1 à $2 \mathrm{~mm}$. de longueur. Dans un cas, cependant, nous avons trouvé des cheveux cassés de $1 \mathrm{~cm}$. de longueur.

Aucun des malades n'a été soumis à un examen en lumière de Wood. Cependant, tous les prélèvements ont été examinés au laboratoire deux ou trois mois après leur prélèvement : aucun ne présentait de fluorescence.

Cas $n^{\circ} 1$. - Garçon âgé de 1 mois $1 / 2$, du village de Kuryange. Présente une plaque pariétale droite de $2 \mathrm{~cm}$. de diamètre dont l'aspect clinique correspond à celui d'une trichophytie. Le prélèvement mis en culture donne une souche (Rv. 8289) de T. kuryangei. A un frère âgé de 7 ans infecté par le même dermatophyte (cas suivant) eì une sœur cliniquement indemne.

Cas $n^{\circ}$ 2. - Frère du précédent, âgé de 7 ans, du village de Kuryange. Diagnostic clinique : trichophytie. Culture : T. kuryangei (Rv. 8290).

Cas $n^{\circ}$ 3. - Garçon de 10 ans, du village de Kuryange. Diagnostic clinique : trichophytie. Culture: T. liuryangei (Rv. 8284) et $T$. violaceum.

Cas $n^{\circ}$ 4. - Garçon de 5 ans, du village de Kuryange. Diagnostic clinique : trichophytie. Culture : T. kuryangei (Rv. 8280) et T. violaceum.

Cas $n^{\circ} 5$. - Femme de 45 ans, du village de Kuryange. Le diagnostic clinique est hésitant : la malade présente quelques plaques glabres d'un demi-centimètre de diamètre, éparses sur la tête. Elle prétend avoir cette maladie depuis toujours. Culture: T. kuryangei (Rv. 8297) et T. violaceum.

Cas $n^{\circ}$ 6. - Femme âgće de 40 ans, du village de Kayanza. Présente une plaque squameuse de $1 \mathrm{~cm}$. de diamètre dans la nuque et au sommet de la tête une cicatrice lisse. Culture : T. kuryangei (Rv. 8427).

Cas $n^{\circ}$ 7. - Fille âgée de 4 ans $1 / 2$, du village de Kayanza. Présente trois petites lésions au sommet de la tête et de la région pariétale gauche.

Cas $n^{\circ}$ 8. - Garçon de 12 ans, de la ville d'Usumbura. Présente dans la région pariétale droite quatre plaques arrondies de 3 à $1 \mathrm{~cm}$. de diamètre. Culture : T. kuryangei (Rv. 8416).

\section{Morphologie macroscopique des cultures}

Sur milieu de Sabouraud (néopeptone Difco $1 \%$, glucose $2 \%$, agar agar $2 \%$, eau de robinet q.s. $\%$ ) à $25^{\circ} \mathrm{C}$, le développement des colonies est assez rapide. Après une dizaine de jours, elles atteignent 8 à $12 \mathrm{~mm}$. de diamètre, sont légèrement duveteuses et parfaitement circulaires. La couleur de la face aérienne de ces colonies est très particulière : les $2 / 3$ de la colonie sont en effet de couleur saumon, tandis que la périphérie est jaune clair. Le verso est jaune clair à chamois. La surface présente un centre légèrement surélevé d'où 
partent des rayons légèrement surélevés vers la périphérie : ces rayons se voient mieux encore au verso de la colonie. La surface est couverte d'un duvet très court et le centre présente souvent des filaments corémiés. La consistance de la colonie est assez élastique et la gélose n'est pas altérée par son développement. Les colonies sont profondément immergées dans la gélose. Les huit souches isolées avaient des caractères identiques. Ce dermatophyte évolue rapidement vers le pléomorphisme. Microscopiquement toutefois, même dans des cultures blanches et duveteuses, on retrouve fréquemment des aleuries.

\section{Morphologie microscopique des cultures}

On distingue nettement un mycélium végétatif fort peu flexueux et un mycélium aérien portant des aleuries. Le mycélium aérien, plus mince et divisé en segments plus courts que le mycélium végétatif, orte des aleuries piriformes disposées suivant le type acladium. Ces aleuries sont généralement assez longues. Parfois, elles peuvent s'allonger considérablement et

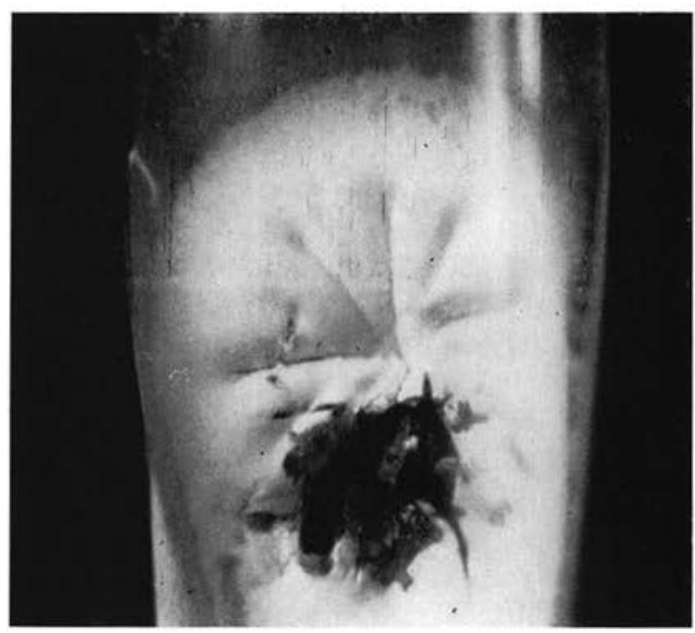

1) Trichophyton kuryangei n. sp. Culture adulte sur Sabouraud se diviser par deux ou trois septa, réalisant ainsi de fausses macroconidies allongées, à parois lisses, qui nous ont fait croire un moment que nous avions affaire à $T$. rubrum. La longueur moyenne des microconidies, établie par la mensuration de 100 d'entre elles prises au hasard, égale $4,19 \mu$, la longueur minimale étant de $1,34 \mu$ et la maximale de $13,4 \mu$. La largeur des microconidies varie de 1,5 à $2,5 \mu$. De plus, les aleuries restent souvent attachées à un segment du mycélium aérien dans lequel le protoplasme s'est concentré, formant ainsi des images en T ou en L. Enfin le mycélium aérien, au lieu de disparaître à la maturité des aleuries, se divise souvent en arthro- 
spores : celles-ci, devenues libres, peuvent aussi simuler des fuseaux de $T$. rubrum. On trouve en outre d'assez nombreuses chlamydospores terminales ou intercalaires.

\section{Morphologie parasitaire chez l'homme}

Dans le cheveu humain, le parasite peut se présenter de différentes façons :

a) soit au début de l'infection, sous forme de filaments intrapilaires ;

b) sous forme d'un dermatophyte endothrix à grosses spores de 5 à $6 \mu$ de diamètre bourrant complètement le cheveu. Cet aspect ne se rencontre qu'à une certaine distance de l'orifice du follicule pileux ;

c) en dessous de l'orifice pileux - peut-être un peu au-dessus le dermatophyte prend l'allure d'un champignon endo-ectothrix, c'est-à-dire qu'on voit le cheveu entouré d'une gaine parfaite de grosses spores - au point de suggérer à un œil non averti une microsporie - et des spores qui le remplissent. Cet aspect correspond à celui qui a été reconnu par Vanbreuseghem (1949) au T. rubrum et au $T$. rodhaini (1949) lorsqu'ils parasitent le cheveu humain ou le poil de l'animal d'expérience. Il se rencontre également dans le parasitisme par $T$. violaceum (Vanbreuseghem, non publié).

La grandeur des spores est le plus souvent de $5 \mu$, mais nous avons vu plus rarement des spores de 3 ou de 4 ou de $6 \mu$ de diamètre.

\section{Morphologie parasitaire chez l'animal d'expérience}

Trois cobayes furent inoculés respectivement avec trois souches différentes de T. kuryangei par la méthode de Rivalier (1926). Deux animaux restèrent indemnes. Le troisième, cependant, présenta deux semaines après l'inoculation des croûtes et de la desquamation. L'examen en lumière de Wood resta négatif, mais les poils présentèrent des filaments intrapilaires et de rares spores ectothrix de 3 à $4 \mu$ de diamètre.

\section{Cultures sur cheveux in vitro}

Huit souches de T. kuryangei furent ensemencées sur cheveux, tant par la technique de Vanbreuseghem (1952) que par celle de L. Ajello et L. K. Georg (1957). Trois des huit souches produisirent, après trois semaines, des organes perforateurs par l'une ou l'autre technique. Six souches de T. rubrum ensemencées semblablement ne produisirent aucun organe perforateur. 


\section{Besoins essentiels}

Dans le but de déterminer leurs besoins vitaminiques, huit souches de T. kuryangei furent cultivées sur trois milieux différents : milieu de base, renfermant de la caséine sans vitamines (L. K. Georg et L. B. Camp 1957) ; milieu de base additionné de 12 vitamines ${ }^{*}$ ) différentes (acide folique, biotine, chlorure de choline, panthoténate de calcium, lactoflavine, adermine, vitamine B12, acide paramino-benzoïque, inositol, thiamine, acide ascorbique, l'amide de l'acide nicotinique) et milieux à l'extrait de levure. Le développement sur les trois milieux fut identique, ce qui signifie, cela va de soi, que $T$. $k u$ ryangei est autotrophe du point de vue de ses besoins en vitamines.

Cependant, $T$. kuryangei ne se développe pas sur un milieu qui ne renferme comme source azotée que du nitrate d'ammonium (L. K. Georg et L. B. Camp 1957), mais pousse si on ajoute au milieu renfermant le $\mathrm{NH}_{4} \mathrm{NO}_{3}$ de l'histidine à la concentration de 25 microgrammes/ml. (ef. photo).

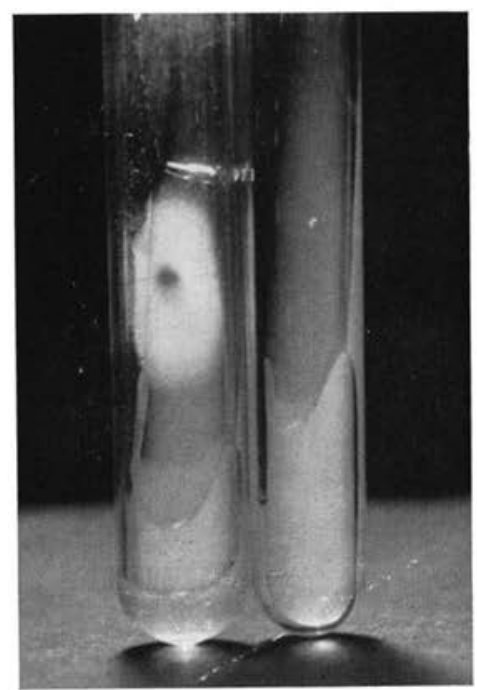

2) Trichophyton kuryangei n'utilise pas le $\mathrm{NH}_{4} \mathrm{NO}_{3}$ (tube de droite), à moins qu'on $\mathrm{y}$ ajoute de l'histidine (tube de gauche).

\section{Caractères différentiels}

Etant donné certaines similitudes entre la morphologie microscopique des cultures de T. kuryangei et celle de T. rubrum, ainsi qu'une similitude accusée de leur morphologie à l'état parasitaire, nous avons cru nécessaire de procéder à certaines comparaisons.

a) Besoins essentiels.

Le $T$. rubrum utilise le $\mathrm{NH}_{4} \mathrm{NO}_{3}$ que n'utilise pas le $T$. kuryangei sans addition d'histidine.

D'autre part, les besoins essentiels de $T$. gallinae sont identiques à ceux de T. kuryangei, presque tous deux sont autotrophes du point de vue de leurs exigences vitaminiques et n'utilisent pas le

(*) Nous remercions le $\mathrm{D}^{\mathrm{r}}$ van den Daele, Directeur de la Maison Roche (Bruxelles), qui a mis généreusement à notre disposition les vitamines employées au cours ae cette expérimentation. 
Nitrate d'Ammonium comme source d'azote, à moins qu'on leur ajoute de l'histidine. Cependant, T. kuryangei ne produit pas de beau pigment rouge diffusant dans la gélose qui caractérise les cultures de T. gallinae.

b) Gulture sur cheveu in vitro.

Le T. rubrum attaque le plus souvent le cheveu selon une modalité décrite par R. Vanbreuseghem, rarement en produisant des organes perforateurs. $T$. kuryangei produit des organes perforateurs (trois de huit souches).

\section{Résumé et conclusions}

Trichophyton kuryangei sp. nov.

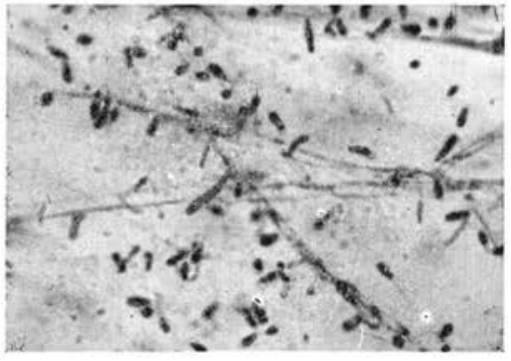

3) a) Microconidies très allongées de Trichophyton kuryangei

Coloniae in agaro Sabouraud orbiculares, plus minus glabrae, in sectoribus divisae radiis superficialibus et a tergo visibilibus. Coloniarum color rosea centrale sed flava ad peripheriam. Coloniae in agaro submersae, a tergo flavae. Sporae $-5 \mu-$ intrapilares atque extrapilares. Aleuriae piriformae plerumque $4,19 \mu \times 1,5-2,5 \mu$ non-nunquam ad $10 \mu$ elongatae macroconidias simulantes. Organos perforatores in pilis in vitro gignit. Chlamydosporae terminales et intercalares. Crescit in agaro a vitaminis privato: $\mathrm{NH}_{4} \mathrm{NO}_{3}$ non utitur nisi cum histidina. Ex tinea capitis, in Ruanda Urundi, Africae.

Le dermatophyte que nous venons de décrire présente les caractères suivants: isolé de teignes trichophytiques du cuir chevelu, il donne lieu sur milieu de Sabouraud au développement de colonies à surface saumon au centre, jaune à la périphérie, légèrement duveteuses, radiées du centre vers la péri-

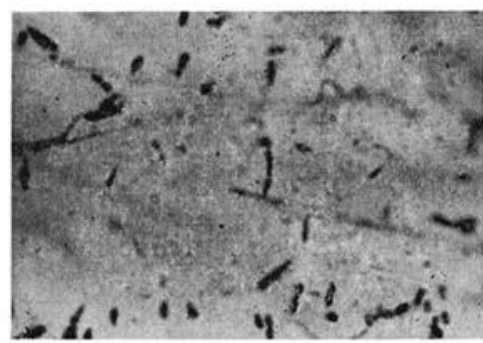

b) Microconidies très allongées de Trichophyton kuryangei phérie. Le verso est chamois et les radiations de la surface s'y voient très bien. Dans le cheveu, il produit une lésion endothrix à grosses 
spores dans la partie distale, endoectothrix dans la partie proximale. Les spores ont $5 \mu$ de diamètre en général. L'élément dominant de la morphologie microscopique est représenté par les aleuries piriformes, parfois allongées au point de simuler des macroconidies septées et des arthrospores libres ou unies aux aleuries. Il y a des chlamydospores. Ce dermatophyte est inoculable au cobaye. Il est autotrophe du point

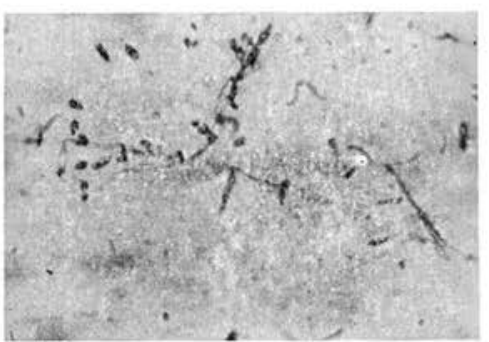

c) Microconidies très allongées de Trichophyton kuryangei

de vue vitaminique, mais ne peut se développer aux dépens du nitrate d'ammonium comme seule source azotée. Il attaque le cheveu in vitro en formant des organes perforateurs.

Pour ces diverses raisons, nous croyons pouvoir le considérer comme un dermatophyte nouveau sous la dénomination de Trichophyton kuryangei n. sp.

\section{RÉFÉRENCES}

Ajello (L.) et Geoni (L. K.), 1957. - - In vitro hair cultures for differentiating between atypical isolates of Trichophyton mentagrophytes and Trichophyton rubrum. Mycopath. et Mycologia Applic., 8, 3, 17.

Georg (L. K.) et Camp (L. B.), 1957. - Routine tests for the identification of Dermatophytes. Jl. Bacteriology, 14, 113-121,

Rivalier (E.), 1926. - Recherches expérimentales sur l'allergie et l'immunité trichophytiques. Ann. Dermat. Syph., 10, 618-640.

Vanbreuseghem (R.), 1949. - A propos de Trichophyton rubrum. Sa présence en Belgique et au Congo Belge. Arch. Belges de Dermat. et Syphiligi., $5 / 4,240-252$.

- 1949. - Contribution à l'étude c'es Dermatophytes du Congo Belge. Deseription du Trichophyton megaspore T. rodhaini n. sp. Ann. de Parasitologie, 24, 3-4, 243-251.

- 1949. - La culture des Dermatophytes $\therefore$ vitro sur des cheveux isolés. Ann. Parasit., 24, 554-573.

1957. - Note préliminaire sur l'endémie teigneuse au Congo Belge et au Ruanda Urundi et ses rapports avec la nutrition. Bull. Acad. Ro!j. Sc. Col., III, 2, 394-410.

1961. - Les teignes du cuir-chevelu au Ruanda Urundi. Ann. Soc. Belge de Méd. Trop., 3, 213-224.

(Travail effectué dans le Laboratoire de Mycologie de IInstitut de Médecine Tropicale à Anvers. Chef du déparlement: $\mathrm{F}^{r} \mathrm{D}^{r}$ VannReuseghem. Directeur de l'Institut: $\mathrm{P}^{\prime}$ P. G. Janssens). 